\section{Effects of Co-medication with Glucocorticoids in Biologics Trials}

To the Editor:

I read with interest the article "Effect of Glucocorticoids on the Clinical and Radiographic Efficacy of Tofacitinib in Patients with Rheumatoid Arthritis: A Posthoc Analysis of Data from 6 Phase III Studies ${ }^{1}$."

I am pleased with the finding of a numerically beneficial effect of current co-treatment with glucocorticoids on radiographic progression in patients treated only with methotrexate (or placebo), because this strengthens earlier observations we made in this journal ${ }^{2}$.

The authors report that the differences between these groups were not statistically significant, but the article was not entirely clear on the analysis strategy. The difference was most apparent at 24 months, but the CI overlapped. Although overlapping CI do not preclude a significant difference $^{3}$, from visual inspection of the figure, the degree of overlap supports the conclusion, and I assume the differences were also formally tested. However, I am not sure whether this difference at 24 months (and the CI) fully incorporated the information on the measurements taken at 6 and 12 months. In other words, did the mixed model analysis result in an overall assessment of the progression rate over 24 months, or were the timepoints tested separately? Optimal power to detect differences is only achieved if the former method was used.

Also, information is missing on the number of patients still taking placebo at 24 months, and on the treatment of the patients who started taking placebo but prematurely discontinued or were switched to active (or other) treatment per protocol. This information would help to further interpret the findings of this posthoc analysis.

MAARTEN BOERS (1), MSc, MD, PhD, Professor of Clinical Epidemiology, Department of Epidemiology and Biostatistics, Amsterdam Rheumatology and Immunology Center, VU University Medical Center, PO Box 7057, 1007 MB Amsterdam, the Netherlands.E-mail: m.boers@VUMC.NL

\section{REFERENCES}

1. Charles-Schoeman C, van der Heijde D, Burmester GR, Nash P, Zerbini CA, Connell CA, et al. Effect of glucocorticoids on the clinical and radiographic efficacy of tofacitinib in patients with rheumatoid arthritis: a posthoc analysis of data from 6 phase III studies. J Rheumatol 2018;45:177-87.

2. Boers M, Aletaha D, Mela CM, Baker DG, Smolen JS

Glucocorticoid effect on radiographic progression in placebo arms of rheumatoid arthritis biologics trials. J Rheumatol 2016; 43:1024-6.

3. Boers M. Null bar and null zone are better than the error bar to compare group means in graphs. J Clin Epidemiol 2004;57:712-5.

J Rheumatol 2018;45:11; doi:10.3899/jrheum.180151 